\title{
THE GLIMM IDEAL SPACE OF A TWO-STEP NILPOTENT LOCALLY COMPACT GROUP
}

\author{
EBERHARD KANIUTH ${ }^{1}$ AND WILLIAM MORAN ${ }^{2}$ \\ ${ }^{1}$ Fachbereich Mathematik/Informatik, Universität Paderborn, \\ D-33095 Paderborn, Germany (kaniuth@uni-paderborn.de) \\ ${ }^{2}$ Department of Mathematics and Statistics, The Flinders University of South \\ Australia, Adelaide 5001, Australia (bill@ist.flinders.edu.au)
}

(Received 18 November 1999)

\begin{abstract}
For a two-step nilpotent locally compact group $G$, we determine the Glimm ideal space of the group $C^{*}$-algebra $C^{*}(G)$ and its topology. This leads to necessary and sufficient conditions for $C^{*}(G)$ to be quasi-standard. Moreover, some results about the Glimm classes of points in the primitive ideal
\end{abstract} space $\operatorname{Prim}\left(C^{*}(G)\right)$ are obtained.

Keywords: two-step nilpotent group; group $C^{*}$-algebra; primitive ideal; Glimm ideal; topology on ideal spaces; quasi-standard $C^{*}$-algebra

AMS 2000 Mathematics subject classification: Primary 22D25

Secondary 22D10

\section{Introduction}

The Glimm ideal space of a $C^{*}$-algebra $A, \operatorname{Glimm}(A)$, arises from the complete regularization $[\mathbf{8}]$ of the primitive ideal space $\operatorname{Prim}(A)$ of $A$. Define an equivalence relation $\sim$ on $\operatorname{Prim}(A)$ by $P \sim Q$ if $f(P)=f(Q)$ for all (bounded) continuous functions $f$ on $\operatorname{Prim}(A)$. Denoting by $[P]$ the equivalence class of $P$, we obtain a bijection between the quotient space $\operatorname{Prim}(A) / \sim$ and the set of Glimm ideals, given by the assignment

$$
[P] \rightarrow k([P])=\bigcap\{Q: Q \in[P]\} .
$$

$\operatorname{Glimm}(A)$ carries the quotient topology obtained from $\operatorname{Prim}(A)$. Clearly, $\operatorname{Glimm}(A)=$ $\operatorname{Prim}(A)$ precisely when $\operatorname{Prim}(A)$ is Hausdorff.

Apart from being vital to understanding the fine structure of the topology of $\operatorname{Prim}(A)$, the Glimm ideal space of $A$ plays an important role in the problem of representing $A$ as a maximal full algebra of cross-sections with varying fibre algebras over a locally compact Hausdorff space. Indeed, if a $C^{*}$-algebra $A$ is representable as a maximal full algebra of cross-sections over a locally compact Hausdorff space $X$ such that the fibres are so-called primal ideals throughout a dense subset of $X$, then $X$ is canonically homeomorphic to $\operatorname{Glimm}(A)[\mathbf{3}]$. Such $C^{*}$-algebras are called quasi-standard, and it turned out that $A$ is 
quasi-standard if and only if the quotient map from $\operatorname{Prim}(A)$ onto $\operatorname{Glimm}(A)$ is open and every Glimm ideal $I$ of $A$ is primal [3] (that is, there is a net in $\operatorname{Prim}(A)$ converging to every point of the hull $h(I)$ of $I$ ).

In this paper we investigate the Glimm ideal space of the $C^{*}$-algebra $C^{*}(G)$ of a two-step nilpotent locally compact group $G$. Firstly, we establish a parametrization of $\operatorname{Glimm}\left(C^{*}(G)\right)$ through triples $\left(\lambda, F_{\lambda}, \tau\right)$, where $\lambda$ is a character of the centre $Z(G), F_{\lambda}$ is a closed subgroup of $G$ associated to $\lambda$, and $\tau$ is a $G$-invariant character of $F_{\lambda}$ extending $\lambda$, and we describe the topology on $\operatorname{Glimm}\left(C^{*}(G)\right)$ in terms of this parametrization (Theorem 3.7). We proceed to characterize openness of the mapping from $\operatorname{Prim}\left(C^{*}(G)\right)$ onto $\operatorname{Glimm}\left(C^{*}(G)\right)$ by means of the mapping $\lambda \rightarrow F_{\lambda}$ (Theorem 4.2), and thereby also deduce necessary and sufficient conditions for $C^{*}(G)$ to be quasi-standard (Corollary 4.4).

In general, it seems to be a difficult problem to determine the Glimm class $[P]$ of a primitive ideal $P$, even for $P$ the kernel of the trivial representation $1_{G}$. In $\S 5$ we add some partial results, including a description of $\left[\right.$ ker $1_{G}$ ] under the assumption that the closure of the commutator subgroup of $G$ has a maximal compact subgroup (Theorem 5.5). Finally, in $\S 6$ we present some examples for which the Glimm ideal space can be computed explicitly.

\section{Preliminaries}

We first recall some notation and basic facts from representation theory. As is customary, we shall use the same letter, for example $\pi$, to denote a unitary representation of a locally compact group $G$ and the associated $*$-representation of the group $C^{*}$-algebra $C^{*}(G)$. Then ker $\pi$ will denote the $C^{*}$-kernel of $\pi$, and $\pi \rightarrow \operatorname{ker} \pi$ defines a mapping from the dual space $\hat{G}$ of $G$ onto $\operatorname{Prim}\left(C^{*}(G)\right)$. If $S$ and $T$ are sets of unitary representations of $G$, then $S$ is weakly contained in $T(S \prec T)$ if $\cap\{\operatorname{ker} \sigma: \sigma \in S\} \supseteq \cap\{\operatorname{ker} \tau: \tau \in T\}$, and $S$ and $T$ are weakly equivalent $(S \sim T)$ if $S \prec T$ and $T \prec S$ (see [10] and [12]).

Throughout the paper $Z(G)$ (or simply $Z$ ) will denote the centre of $G$. Then, for an irreducible representation $\pi$ of $G, \pi \mid Z \sim \lambda_{\pi}$ for a unique character $\lambda_{\pi} \in \hat{Z}$, and $\pi \sim \rho$ implies that $\lambda_{\pi}=\lambda_{\rho}$. This defines a mapping

$$
r: \operatorname{Prim}\left(C^{*}(G)\right) \rightarrow \hat{Z}, \quad \operatorname{ker} \pi \rightarrow \lambda_{\pi},
$$

which is well known to be continuous and surjective. For a closed subgroup $H$ of $G$ and a representation $\tau$ of $H, \operatorname{ind}_{\mathrm{H}}^{G} \tau$ denotes the representation of $G$ induced by $\tau$. Then, for any representation $\pi$ of $G, \pi \otimes \operatorname{ind}_{\mathrm{H}}^{G} \tau=\operatorname{ind}_{\mathrm{H}}^{G}(\pi \mid H \otimes \tau)$, with $\otimes$ denoting the tensor product of representations. The following two simple lemmas will be frequently used in the sequel in the special case where $G$ is two-step nilpotent and $N$ contains the centre.

Lemma 2.1. Let $G$ be an arbitrary locally compact group and $N$ a closed normal subgroup of $G$ with abelian quotient group $G / N$. Then the assignment $(\chi, \operatorname{ker} \pi) \rightarrow$ $\operatorname{ker}(\pi \otimes \chi)$ for $\pi \in \hat{G}$ and $\chi \in \widehat{G / N}$ defines a continuous mapping $(\chi, P) \rightarrow \chi \cdot P$ from $\widehat{G / N} \times \operatorname{Prim}\left(C^{*}(G)\right)$ onto $\operatorname{Prim}\left(C^{*}(G)\right)$. 
Proof. Clearly, $\pi \otimes \chi \in \hat{G}$ for $\pi \in \hat{G}$ and $\chi \in \widehat{G / N}$ and $\pi \otimes \chi \sim \rho \otimes \chi, \rho \in \hat{G}$, implies $\pi \sim \rho$. Thus $\Phi:(\chi, P) \rightarrow \chi \cdot P$ is well defined. Similarly, we have a mapping

$$
\Psi: \widehat{G / N} \times \hat{G} \rightarrow \hat{G}, \quad(\chi, \pi) \rightarrow \pi \otimes \chi .
$$

Since coordinate functions $\varphi$ of $\pi \otimes \chi$ are of the form

$$
\varphi(x)=\langle(\pi \otimes \chi)(x) \xi, \eta\rangle=\chi(x)\langle\pi(x) \xi, \eta\rangle,
$$

where $\xi, \eta \in \mathcal{H}_{\pi \otimes \chi}=\mathcal{H}_{\pi}$, it follows from $[\mathbf{1 0}, \mathbf{1 8}]$ that the map $\Psi$ is continuous. Now, consider the commutative diagram

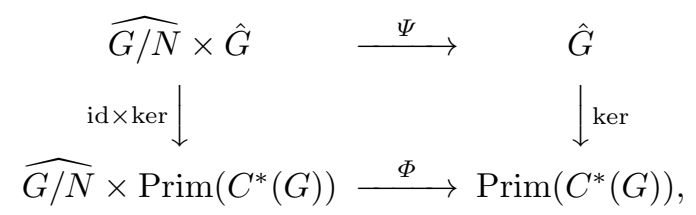

where ker is the kernel map sending $\pi$ to $\operatorname{ker} \pi$. Since $\Psi$ is continuous and the topology on $\hat{G}$ is the inverse image of the hull-kernel topology on $\operatorname{Prim}\left(C^{*}(G)\right)$ under ker, we conclude that $\Phi$ is continuous.

Lemma 2.2. Let $H$ and $N$ be closed subgroups of $G$ such that $N$ is normal and $G / N$ is abelian, and suppose that $\alpha$ is a $G$-invariant character of $N$. Then

$$
\left.\operatorname{(ind}_{N}^{G} \alpha\right) \mid H \sim \operatorname{ind}_{H \cap N}^{H}(\alpha \mid H \cap N) .
$$

Proof. The set of all characters $\sigma \mid H$, where $\sigma \in \widehat{G / N}$, forms a subgroup of the dual group $H \widehat{/ H \cap} N$ which separates the points of $H / H \cap N$ and hence is dense in $H \widehat{H H \cap} N$. Since $\alpha$ is $G$-invariant, there exists a representation $\pi$ of $G$ such that $\pi \mid N \sim \alpha$ (for instance, $\pi=\operatorname{ind}_{N}^{G} \alpha$ ). Weak containment is preserved under tensor products, so that

$$
\begin{aligned}
\left.\operatorname{(ind}_{N}^{G} \alpha\right) \mid H & \sim\left(\operatorname{ind}_{N}^{G}(\pi \mid N)\right)\left|H=\left(\pi \otimes \operatorname{ind}_{N}^{G} 1_{N}\right)\right| H=\pi\left|H \otimes\left(\operatorname{ind}_{N}^{G} 1_{\mathrm{H}}\right)\right| H \\
& \sim\{\pi|H \otimes \sigma| H: \sigma \in \widehat{G / N}\} \sim\{\pi \mid H \otimes \tau: \tau \in H \widehat{H \cap N}\} \\
& \sim \operatorname{ind}_{H \cap N}^{H}(\pi \mid H \cap N) \sim \operatorname{ind}_{H \cap N}^{H}(\alpha \mid H \cap N) .
\end{aligned}
$$

For a locally compact group $G$, let $\mathcal{K}(G)$ be the set of all closed subgroups of $G$ and $\mathcal{S}(G)$ the set of all pairs $(H, \tau)$, where $H \in \mathcal{K}(G)$ and $\tau$ is a unitary representation of $G$. Endow $\mathcal{K}(G)$ with the compact-open topology [11] and $\mathcal{S}(G)$ with Fell's so-called subgroup representation topology [12]. For details concerning the latter compare $\S 3$ of [12], in particular Theorem 3.1' and the remark following it. Both $\mathcal{K}(G)$ and $\mathcal{S}(G)$ are compact. The projection $(H, \tau) \rightarrow H$ from $\mathcal{S}(G)$ onto $\mathcal{K}(G)$ is continuous. The properties that will be most useful to us are continuity of restriction and continuity of induction. 
Theorem 2.3 (see [12]). Let $\mathcal{W}_{1}=\{(F, H, \tau): F \in \mathcal{K}(G),(H, \tau) \in \mathcal{S}(G), F \subseteq H\}$ and $\mathcal{W}_{2}=\{(F, H, \tau): F \in \mathcal{K}(G),(H, \tau) \in \mathcal{S}(G), F \supseteq H\}$.

(i) The mapping $(F, H, \tau) \rightarrow(F, \tau \mid F)$ from $\mathcal{W}_{1}$ into $\mathcal{S}(G)$ is continuous.

(ii) The mapping $(F, H, \tau) \rightarrow\left(F, \operatorname{ind}_{\mathrm{H}}^{F} \tau\right)$ from $\mathcal{W}_{2}$ into $\mathcal{S}(G)$ is continuous.

For instance, (ii) implies that if $\left(\left(H_{\alpha}, \tau_{\alpha}\right)\right)_{\alpha}$ is a net in $\mathcal{S}(G)$ converging to $(H, \tau)$ and $\rho$ is an irreducible representation of $G$ that is weakly contained in $\operatorname{ind}_{\mathrm{H}}^{G} \tau$, then, after passing to a subnet if necessary, we can assume that there are $\rho_{\alpha} \in \hat{G}$ such that $\rho_{\alpha} \prec \operatorname{ind}_{H_{\alpha}}^{G} \tau_{\alpha}$ and $\rho_{\alpha} \rightarrow \rho$ in $\hat{G}$.

Now, let $G$ be a two-step nilpotent locally compact group with centre $Z$. For $\lambda \in \hat{Z}$, let $K_{\lambda}=\{z \in Z: \lambda(z)=1\}$ and $G_{\lambda}$ the preimage in $G$ of the centre of $G / K_{\lambda}$. Let $\varphi$ be a character of $G_{\lambda}$ with $\varphi \mid Z=\lambda$. Then $\operatorname{ker}\left(\operatorname{ind}_{G_{\lambda}}^{G} \varphi\right)$ is a primitive ideal of $C^{*}(G)$, and every $P \in \operatorname{Prim}\left(C^{*}(G)\right)$ is obtained in this way. More precisely, denote by $\mathcal{P}$ the set of all triples $\left(\lambda, G_{\lambda}, \varphi\right)$, where $\lambda \in \hat{Z}$ and $\varphi \in \hat{G}_{\lambda}$ such that $\varphi \mid Z=\lambda$. Then we have the following lemma (see [15, Proposition 5] and [17, Lemma 2]).

Lemma 2.4. The map $\left(\lambda, G_{\lambda}, \varphi\right) \rightarrow \operatorname{ker}\left(\operatorname{ind}_{G_{\lambda}}^{G} \varphi\right)$ is a one-to-one correspondence between $\mathcal{P}$ and $\operatorname{Prim}\left(C^{*}(G)\right)$.

In [6], for $G$ a second-countable two-step nilpotent locally compact group, Baggett and Packer described the hull-kernel topology on $\operatorname{Prim}\left(C^{*}(G)\right)$ in terms of the parameter space $\mathcal{P}$ and Fell's [12] subgroup representation topology on the set $\mathcal{S}(G)$ of all pairs $(H, \tau)$, where $H \in \mathcal{K}(G)$ (the set of all closed subgroups of $G$ ) and $\tau$ is a representation of $H$. In so doing they used the existence of a Borel cross-section for $G / Z$ and cocycles on $G / Z$. However, this can be avoided, as can the assumption that $G$ be second countable. In what follows we restate their result in the general case and give the necessary modifications. It is easy to check that the following definition of convergence defines a topology on $\mathcal{P}$.

Definition 2.5. A net $\mathcal{N}$ in $\mathcal{P}$ converges to $\left(\lambda, G_{\lambda}, \varphi\right) \in \mathcal{P}$ if every subnet $\mathcal{N}^{\prime}$ of $\mathcal{N}$ contains in turn a subnet $\left(\lambda_{\alpha}, G_{\lambda_{\alpha}}, \varphi_{\alpha}\right)_{\alpha}$ such that

(i) $\lambda_{\alpha} \rightarrow \lambda$ in $\hat{Z}$, and

(ii) $\left(G_{\lambda_{\alpha}}, \varphi_{\alpha}\right)$ converges to some $(H, \psi)$ in $\mathcal{S}(G)$ with $H \subseteq G_{\lambda}$ and $\varphi \mid H=\psi$.

Theorem 2.6 (see Theorem 1.6 in [6]). Let $G$ be a two-step nilpotent locally compact group. With the topology defined as in Definition 2.5, the mapping

$$
\left(\lambda, G_{\lambda}, \varphi\right) \rightarrow \operatorname{ker}\left(\operatorname{ind}_{G_{\lambda}}^{G} \varphi\right)
$$

is a homeomorphism between $\mathcal{P}$ and $\operatorname{Prim}\left(C^{*}(G)\right)$. 
In a similar way, the topology of $\operatorname{Prim}\left(C^{*}(G)\right)$ was previously described for certain semi-direct product groups in [5]. This study, in turn, was extended to a wider class of semi-direct product groups by Williams $[\mathbf{2 3}]$.

Carefully checking the proof of Theorem 2.6 as given in [6] for second-countable $G$, one observes that all arguments except one carry over to the non-second-countable case when nets are substituted for sequences. The exception is when cross-sections and cocycles are employed to show that if $\left(\lambda_{\alpha}\right)_{\alpha}$ is a net in $\hat{Z}$ such that $\lambda_{\alpha} \rightarrow \lambda$ in $\hat{Z}$ and $G_{\lambda_{\alpha}} \rightarrow F$ in $\mathcal{K}(G)$, then $F \subseteq G_{\lambda}$. However, this can be seen as follows. Given $x \in F$, after moving to a subnet if necessary, we can assume that there are $x_{\alpha} \in G_{\lambda_{\alpha}}$ so that $x_{\alpha} \rightarrow x[\mathbf{1 1}]$. Then, denoting by $[a, b]$ the commutator of $a$ and $b$, we have $\left[x_{\alpha}, y\right] \rightarrow[x, y]$ for every $y \in G$ and $\lambda_{\alpha}\left(\left[x_{\alpha}, y\right]\right)=1$ for all $\alpha$. Since $(\mu, z) \rightarrow \mu(z)$ is a continuous function on $\hat{Z} \times Z$, we conclude that $\lambda([x, y])=1$ for every $y \in G$, whence $x \in G_{\lambda}$.

\section{The Glimm ideal space of $C^{*}(G)$}

Let $G$ be a two-step nilpotent locally compact group. The aim of this section is to find an appropriate parametrization of $\operatorname{Glimm}\left(C^{*}(G)\right)$ and to describe the topology on $\operatorname{Glimm}\left(C^{*}(G)\right)$ in terms of this parametrization and convergence of nets. We continue to denote by $Z$ the centre of $G$.

Lemma 3.1. Given $\lambda \in \hat{Z}$, there exists a smallest closed subgroup $F_{\lambda}$ of $G$ (containing $Z)$ such that $[P]=\widehat{G / F_{\lambda}} \cdot P$ for every $P \in r^{-1}(\lambda)$.

Proof. Since every continuous function on $\hat{Z}$ composed with $r$ defines a continuous function on $\operatorname{Prim}\left(C^{*}(G)\right)$, we have that $[P] \subseteq r^{-1}(\lambda)$ for each $P \in r^{-1}(\lambda)$. Also, recall that $r^{-1}(\lambda)=\widehat{G / Z} \cdot P$.

Fix $P \in r^{-1}(\lambda)$, and let $X_{P}=\{\chi \in \widehat{G / Z}: \chi \cdot P \in[P]\}$. Then $X_{P}$ is a closed subgroup of $\widehat{G / Z}$. To see this, notice first that since the mapping

$$
\widehat{G / Z} \times \operatorname{Prim}\left(C^{*}(G)\right) \rightarrow \operatorname{Prim}\left(C^{*}(G)\right), \quad(\chi, Q) \rightarrow \chi \cdot Q
$$

is continuous, $X_{P}$ is closed in $\widehat{G / Z}$, and we can associate to every continuous function $f$ on $\operatorname{Prim}\left(C^{*}(G)\right)$ and $\chi \in \widehat{G / Z}$ a new continuous function $g$ by setting $g(Q)=f(\chi \cdot Q)$. It follows that, if $\chi, \rho \in X_{P}$, then

$$
f((\chi \rho) \cdot P)=g(\rho \cdot P)=g(P)=f(\chi \cdot P)=f(P),
$$

and, similarly, $f\left(\chi^{-1} \cdot P\right)=f(P)$. Thus $X_{P}$ is a closed subgroup of $\widehat{G / Z}$ and hence $X_{P}=\widehat{G / F_{P}}$ for a unique closed subgroup $F_{P}$ of $G$, and $F_{P} \supseteq Z$. If $H$ is an arbitrary closed subgroup of $G$, containing $Z$, such that $[P]=\widehat{G / H} \cdot P$, then $\widehat{G / H} \subseteq X_{P}$, whence $H \supseteq F_{P}$. 
Finally, if $Q \in r^{-1}(\lambda)$ and hence $Q=\rho \cdot P$ for some $\rho \in \widehat{G / Z}$, then

$$
\begin{aligned}
X_{Q} & =\left\{\chi \in \widehat{G / Z}: f(\rho \cdot(\chi \cdot P))=f(\rho \cdot P) \text { for all } f \in C\left(\operatorname{Prim}\left(C^{*}(G)\right)\right)\right\} \\
& =\left\{\chi \in \widehat{G / Z}: g(\chi \cdot P)=g(P) \text { for all } g \in C\left(\operatorname{Prim}\left(C^{*}(G)\right)\right)\right\}=X_{P} .
\end{aligned}
$$

Thus $F_{Q}=F_{P}$ for all $Q \in r^{-1}(\lambda)$ and we can unambiguously set $F_{\lambda}=F_{P}$.

Obviously, $F_{\lambda} \subseteq G_{\lambda}$ for every $\lambda \in \hat{Z}$. Let $\mathcal{G}$ denote the set of all (Glimm) triples $\left(\lambda, F_{\lambda}, \tau\right)$, where $\lambda \in \hat{Z}$ and $\tau$ is a character of $F_{\lambda}$ such that $\tau \mid Z=\lambda$.

Corollary 3.2. The map $\left(\lambda, F_{\lambda}, \tau\right) \rightarrow \operatorname{ker}\left(\operatorname{ind}_{F_{\lambda}}^{G} \tau\right)$ is a bijection between $\mathcal{G}$ and $\operatorname{Glimm}\left(C^{*}(G)\right)$.

Proof. Let $\lambda \in \hat{Z}$ and $\varphi \in \hat{G}_{\lambda}$ be such that $\varphi \mid Z=\lambda$, and let $P=\operatorname{ker}\left(\operatorname{ind}_{G_{\lambda}}^{G} \varphi\right)$. Then

$$
k([P])=\operatorname{ker}\left(\operatorname{ind}_{F_{\lambda}}^{G}\left(\varphi \mid F_{\lambda}\right)\right) .
$$

Indeed, since $\widehat{G_{\lambda} / F_{\lambda}} \cdot \varphi$ is weakly equivalent to $\operatorname{ind}_{F_{\lambda}}^{G_{\lambda}}\left(\varphi \mid F_{\lambda}\right)$, we obtain from Lemma 3.1 that

$$
\begin{aligned}
k([P]) & =\bigcap\left\{\chi \cdot P: \chi \in \widehat{G / F_{\lambda}}\right\}=\bigcap\left\{\operatorname{ker}\left(\chi \otimes \operatorname{ind}_{G_{\lambda}}^{G} \varphi\right): \chi \in \widehat{G / F_{\lambda}}\right\} \\
& =\bigcap\left\{\operatorname{ker}\left(\operatorname{ind}_{G_{\lambda}}^{G}\left(\chi \mid G_{\lambda} \cdot \varphi\right)\right): \chi \in \widehat{G / F_{\lambda}}\right\}=\bigcap\left\{\operatorname{ker}\left(\operatorname{ind}_{G_{\lambda}}^{G}(\rho \varphi)\right): \rho \in \widehat{G_{\lambda} / F_{\lambda}}\right\} \\
& =\operatorname{ker}\left(\operatorname{ind}_{G_{\lambda}}^{G}\left(\operatorname{ind}_{F_{\lambda}}^{G_{\lambda}}\left(\varphi \mid F_{\lambda}\right)\right)\right)=\operatorname{ker}\left(\operatorname{ind}_{F_{\lambda}}^{G}\left(\varphi \mid F_{\lambda}\right)\right) .
\end{aligned}
$$

This shows that $\left(\lambda, F_{\lambda}, \tau\right) \rightarrow \operatorname{ker}\left(\operatorname{ind}_{F_{\lambda}}^{G} \tau\right)$ maps $\mathcal{G}$ onto $\operatorname{Glimm}\left(C^{*}(G)\right)$. To verify that this mapping is also injective, let $\lambda_{i} \in \hat{Z}$ and $\tau_{i} \in \hat{F}_{\lambda_{i}}, i=1,2$, be such that $\tau_{i} \mid Z=\lambda_{i}$ and the representations $\pi_{1}=\operatorname{ind}_{F_{\lambda_{1}}}^{G} \tau_{1}$ and $\pi_{2}=\operatorname{ind}_{F_{\lambda_{2}}}^{G} \tau_{2}$ have the same kernel in $C^{*}(G)$. Then $\lambda_{1}=\lambda_{2}$ and, since $\tau_{1}$ and $\tau_{2}$ are $G$-invariant characters, it follows that, with $F=F_{\lambda_{1}}=F_{\lambda_{2}}$,

$$
\tau_{1} \sim \pi_{1}\left|F \sim \pi_{2}\right| F \sim \tau_{2}
$$

Thus $\tau_{1}=\tau_{2}$, as required.

We now define a topology on $\mathcal{G}$ which will turn out to make the bijection of Corollary 3.2 a homeomorphism.

Definition 3.3. A net

$$
\left(\lambda_{\alpha}, F_{\lambda_{\alpha}}, \tau_{\alpha}\right)_{\alpha \in A} \subseteq \mathcal{G}
$$

converges to $\left(\lambda, F_{\lambda}, \tau\right)$ in $\mathcal{G}$ if every subnet $\left(\lambda_{\alpha_{\beta}}, F_{\lambda_{\alpha_{\beta}}}, \tau_{\alpha_{\beta}}\right)_{\beta \in B}$ of $\left(\lambda_{\alpha}, F_{\lambda_{\alpha}}, \tau_{\alpha}\right)_{\alpha \in A}$ contains in turn a subnet $\left(\lambda_{\alpha_{\beta_{\gamma}}}, F_{\lambda_{\alpha_{\beta_{\gamma}}}}, \tau_{\alpha_{\beta_{\gamma}}}\right)_{\gamma \in C}$ such that 
(i) $\lambda_{\alpha_{\beta \gamma}} \rightarrow \lambda$ in $\hat{Z}$, and

(ii) $\left(F_{\lambda_{\alpha_{\beta \gamma}}}, \tau_{\alpha_{\beta \gamma}}\right)_{\gamma \in C}$ converges to some $(F, \sigma)$ in $\mathcal{S}(G)$ such that $F \supseteq F_{\lambda}$ and $\sigma \mid F_{\lambda}=$ $\tau$.

It is easy to check that this definition of convergence defines a topology on $\mathcal{G}$. The following lemma is the first step in proving that, with this topology, $\mathcal{G}$ is homeomorphic to $\operatorname{Glimm}\left(C^{*}(G)\right)$.

Lemma 3.4. The map $\mathcal{G} \rightarrow \operatorname{Glimm}\left(C^{*}(G)\right)$ is continuous.

Proof. Let $\mathcal{N}=\left(\lambda_{\alpha}, F_{\lambda_{\alpha}}, \tau_{\alpha}\right)_{\alpha}$ be a net in $\mathcal{G}$ converging to $\left(\lambda, F_{\lambda}, \tau\right)$ in $\mathcal{G}$. Then every subnet of $\mathcal{N}$ contains a subnet $\left(\lambda_{\alpha_{\beta}}, F_{\lambda_{\alpha_{\beta}}}, \tau_{\alpha_{\beta}}\right)_{\beta}$ such that $\lambda_{\alpha_{\beta}} \rightarrow \lambda$ in $\hat{Z}$ and $\left(F_{\lambda_{\alpha_{\beta}}}, \tau_{\alpha_{\beta}}\right)_{\beta} \rightarrow(F, \sigma)$ in $\mathcal{S}(G)$ with $F \supseteq F_{\lambda}$ and $\sigma \mid F_{\lambda}=\tau$. By continuity of inducing,

$$
\operatorname{ind}_{F_{\lambda_{\beta} \beta}}^{G} \tau_{\alpha_{\beta}} \rightarrow \operatorname{ind}_{F}^{G} \sigma .
$$

Choose $P \in h\left(\operatorname{ker}\left(\operatorname{ind}_{F}^{G} \sigma\right)\right)$. Then there exist $P_{\beta} \in h\left(\operatorname{ind}_{F_{\lambda_{\alpha}}}^{G} \tau_{\alpha_{\beta}}\right)$ such that $P_{\alpha_{\beta}} \rightarrow P$ in $\operatorname{Prim}\left(C^{*}(G)\right)$. Since the quotient map $q: \operatorname{Prim}\left(C^{*}(G)\right) \rightarrow \operatorname{Glimm}\left(C^{*}(G)\right)$ is continuous,

$$
\operatorname{ker}\left(\operatorname{ind}_{F_{\lambda_{\alpha} \beta}}^{G} \tau_{\alpha_{\beta}}\right)=q\left(P_{\beta}\right) \rightarrow q(P)=k([P]) .
$$

However, since $\sigma \mid F_{\lambda}=\tau$, we have that $\sigma \prec \operatorname{ind}_{F_{\lambda}}^{F} \tau$ and hence

$$
P \in h\left(\operatorname{ker}\left(\operatorname{ind}_{F}^{G} \sigma\right)\right) \subseteq h\left(\operatorname{ker}\left(\operatorname{ind}_{F_{\lambda}}^{G} \tau\right)\right) .
$$

As $\operatorname{ker}\left(\operatorname{ind}_{F_{\lambda}}^{G} \tau\right)$ is a Glimm ideal, it follows that $\operatorname{ker}\left(\operatorname{ind}_{F_{\lambda}}^{G} \tau\right)=k([P])$. Thus we have seen that every subnet of the net $\left(\operatorname{ker}\left(\operatorname{ind}_{F_{\lambda \alpha}}^{G} \tau_{\alpha}\right)\right)_{\alpha}$ contains a subnet converging to $\operatorname{ker}\left(\operatorname{ind}_{F_{\lambda}}^{G} \tau\right)$. This proves the lemma.

Lemma 3.5. If $\lambda_{\alpha} \rightarrow \lambda$ in $\hat{Z}$ and $F_{\lambda_{\alpha}} \rightarrow F$ in $\mathcal{K}(G)$, then $F \supseteq F_{\lambda}$.

Proof. Choose any $P \in r^{-1}(\lambda)$. Then $[P]=\widehat{G / F_{\lambda}} \cdot P$ by Lemma 3.1. To prove that $F_{\lambda} \subseteq F$, it suffices to show that $\chi \cdot P \in[P]$ for every $\chi \in \widehat{G / F}$ (compare the proof of Lemma 3.1). With this in mind, fix $\chi \in \widehat{G / F}$.

Now, for any locally compact abelian group $A$, by $[\mathbf{2 2}]$ the map $H \rightarrow \widehat{A / H}$ is a homeomorphism between $\mathcal{K}(A)$ and $\mathcal{K}(\hat{A})$. Since $F_{\lambda_{\alpha}} / Z \rightarrow F / Z$ in $\mathcal{K}(G / Z)$, it follows that $\widehat{G / F_{\lambda_{\alpha}}} \rightarrow \widehat{G / F}$ in $\mathcal{K}(\widehat{G / F})$. Therefore, for each $\alpha$, there exists $\chi_{\alpha} \in \widehat{G / F_{\lambda_{\alpha}}}$ such that $\chi_{\alpha} \rightarrow \chi$ in $\widehat{G / Z}$. On the other hand, since $\operatorname{ker}\left(\operatorname{ind}_{Z}^{G} \lambda_{\alpha}\right) \rightarrow P$, for each $\alpha$, we find some $P_{\alpha} \in r^{-1}\left(\lambda_{\alpha}\right)$ such that $P_{\alpha} \rightarrow P$ in $\operatorname{Prim}\left(C^{*}(G)\right)$. Since the mapping

$$
\widehat{G / Z} \times \operatorname{Prim}\left(C^{*}(G)\right) \rightarrow \operatorname{Prim}\left(C^{*}(G)\right), \quad(\sigma, Q) \rightarrow \sigma \cdot Q
$$


is continuous, we conclude that $\chi_{\alpha} \cdot P_{\alpha} \rightarrow \chi \cdot P$ in $\operatorname{Prim}\left(C^{*}(G)\right)$. Now, as $\chi_{\alpha} \cdot P_{\alpha} \in\left[P_{\alpha}\right]$ and hence $f\left(\chi_{\alpha} \cdot P_{\alpha}\right)=f\left(P_{\alpha}\right)$ for every $\alpha$ and every continuous function $f$ on $\operatorname{Prim}\left(C^{*}(G)\right)$, it follows that $f(\chi \cdot P)=f(P)$ for each such $f$, as required.

The preceding lemma will be used to prove the next lemma as well as later in the paper $(\S 4)$.

Lemma 3.6. The map $\mathcal{P} \rightarrow \mathcal{G},\left(\lambda, G_{\lambda}, \varphi\right) \rightarrow\left(\lambda, F_{\lambda}, \varphi \mid F_{\lambda}\right)$ is continuous.

Proof. Let $\mathcal{N}$ be a net in $\mathcal{P}$ converging to some $\left(\lambda, G_{\lambda}, \varphi\right)$ in $\mathcal{P}$, and let $\mathcal{N}^{\prime}$ be a subnet of $\mathcal{N}$. Then $\mathcal{N}^{\prime}$ possesses a subnet $\left(\lambda_{\alpha}, G_{\lambda_{\alpha}}, \varphi_{\alpha}\right)_{\alpha}$ such that $\lambda_{\alpha} \rightarrow \lambda$ in $\hat{Z}$ and $\left(G_{\lambda_{\alpha}}, \varphi_{\alpha}\right) \rightarrow(H, \psi)$ in $\mathcal{S}(G)$ with $H \subseteq G_{\lambda}$ and $\psi=\varphi \mid H$.

Since $\mathcal{K}(G)$ is compact, by passing to a further subnet if necessary, we can assume that $F_{\lambda_{\alpha}} \rightarrow F$ in $\mathcal{K}(G)$. As $F_{\mu} \subseteq G_{\mu}$ for all $\mu \in \hat{Z}$, it follows that $F \subseteq H$ and hence $\left(F_{\lambda_{\alpha}}, \varphi_{\alpha} \mid F_{\lambda_{\alpha}}\right) \rightarrow(F, \varphi \mid F)$ in $\mathcal{S}(G)$. On the other hand, $F \supseteq F_{\lambda}$ by Lemma 3.5 .

Thus, with $p: \mathcal{P} \rightarrow \mathcal{G}$ denoting the map $\left(\lambda, G_{\lambda}, \rho\right) \rightarrow\left(\lambda, F_{\lambda}, \rho \mid F_{\lambda}\right)$, we have seen that the subnet $p\left(\mathcal{N}^{\prime}\right)$ of $p(\mathcal{N})$ contains a subnet converging in $\mathcal{G}$ to $p\left(\lambda, G_{\lambda}, \varphi\right)$. This proves that $p$ is continuous.

We are now ready to establish the main result of this section.

Theorem 3.7. Let $G$ be a two-step nilpotent locally compact group. With the topology on $\mathcal{G}$ as defined in Definition 3.3, the map from $\mathcal{G}$ to $\operatorname{Glimm}\left(C^{*}(G)\right)$ given by $\left(\lambda, F_{\lambda}, \tau\right) \rightarrow \operatorname{ker}\left(\operatorname{ind}_{F_{\lambda}}^{G} \tau\right)$ is a homeomorphism.

Proof. Denote this map by $\Gamma$. Then $\Gamma$ is continuous by Lemma 3.4. In order to prove the openness of $\Gamma$, consider the following commutative diagram, where $q$ is the quotient map, $p$ is the map of Lemma 3.6 and $\Pi: \mathcal{P} \rightarrow \operatorname{Prim}\left(C^{*}(G)\right)$ the parametrization map of Theorem 2.6:

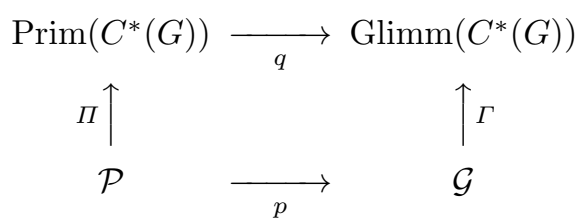

For an open subset $V$ of $\mathcal{G}, q^{-1}(\Gamma(V))=\Pi\left(p^{-1}(V)\right)$ is open since $p$ is continuous (Lemma 3.6) and $\Pi$ is open (Theorem 2.6). Since $\operatorname{Glimm}\left(C^{*}(G)\right)$ carries the quotient topology, it follows that $\Gamma(V)$ is open.

In $\S 6$ we will present examples illustrating Theorem 3.7, Heisenberg groups over the $p$-adic numbers on the one hand and universal two-step nilpotent simply connected Lie groups on the other. We conclude this section with a remark concerning the Glimm ideal space of discrete groups [18]. 
Remark 3.8. Let $G$ be an amenable discrete group, and let $\mathrm{FC}(G)$ denote the normal subgroup consisting of all elements of $G$ with finite conjugacy classes. Let $E(\operatorname{FC}(G), G)$ be the set of all indecomposable normalized $G$-invariant (with respect to conjugation) positive definite functions on $\operatorname{FC}(G)$, endowed with the topology of pointwise convergence. For $\varphi \in E(\operatorname{FC}(G), G)$, denote by $\pi_{\varphi}$ the Gelfand-Naimark-Segal representation of $\operatorname{FC}(G)$ associated with $\varphi$. Then the mapping $\varphi \rightarrow \operatorname{ker}\left(\operatorname{ind}_{\mathrm{FC}(G)}^{G} \pi_{\varphi}\right)$ is a homeomorphism between $E(\operatorname{FC}(G), G)$ and $\operatorname{Glimm}\left(C^{*}(G)\right)[\mathbf{1 8}$, Theorem 3.6]. In particular, if $G$ is a torsion-free nilpotent discrete group, then $\operatorname{FC}(G)$ equals the centre $Z$ of $G$ and hence $\operatorname{Glimm}\left(C^{*}(G)\right)$ is naturally isomorphic to the dual group $\hat{Z}$. However, the family of amenable discrete groups (more generally, amenable groups with small invariant neighbourhoods of the identity) is the only larger class of groups, for which such a complete description of the Glimm ideal space is known.

\section{Quasi-standardness}

A $C^{*}$-algebra $A$ is said to be quasi-standard if the failure of separation by open sets defines an open equivalence relation on $\operatorname{Prim}(A)$. This condition is a natural substitute for the stronger condition that $\operatorname{Prim}(A)$ should be Hausdorff. In the separable case, quasistandardness of $A$ is equivalent to $A$ being representable as a maximal full algebra of cross-sections over a locally compact Hausdorff space, namely Glimm $(A)$, such that the fibres are primitive throughout a dense subset [3]. By [3, Theorem 3.3], a $C^{*}$-algebra $A$ is quasi-standard if and only if the quotient map from $\operatorname{Prim}(A)$ onto $\operatorname{Glimm}(A)$ is open and every Glimm ideal $I$ in $A$ is primal, that is, there exists a net $\left(P_{\alpha}\right)_{\alpha}$ in $\operatorname{Prim}(A)$ such that $P_{\alpha} \rightarrow P$ for each $P \in h(I)$.

The $C^{*}$-algebras of the continuous and the discrete Heisenberg groups have been known to be quasi-standard for some time (see $[\mathbf{1}]$ and $[\mathbf{1 9}]$ ). A more systematic study of quasistandardness of group $C^{*}$-algebras was only recently undertaken in [18], [2] and [4]. $C^{*}(G)$ turned out to be quasi-standard for every amenable discrete group $G[\mathbf{1 8}]$, while for a two-step nilpotent simply connected Lie group $G, C^{*}(G)$ is quasi-standard if and only if the maximal coadjoint orbit dimension in the dual vector space of the Lie algebra of $G$ equals the dimension of $G / Z$ (see [2]).

In this section we are going to characterize, for an arbitrary two-step nilpotent locally compact group $G$, openness of the quotient map and the property that every Glimm is primal in terms of the maps $\lambda \rightarrow F_{\lambda}$ and $\lambda \rightarrow G_{\lambda}$ from $\hat{Z}$ into $\mathcal{K}(G)$, respectively.

Lemma 4.1. Let $\mu \in \hat{Z}$, and let $L$ be a closed subgroup of $G$ such that $Z \subseteq L \subseteq G_{\mu}$. Then $\widehat{G / L} \cdot I$ is closed in $\operatorname{Prim}\left(C^{*}(G)\right)$ for every $I \in r^{-1}(\mu)$.

Proof. Let $\left(\chi_{\alpha} \cdot I\right)_{\alpha}$ be a net in $\widehat{G / L} \cdot J$ converging to some $J \in \operatorname{Prim}\left(C^{*}(G)\right)$. Then $J \in r^{-1}(\mu)$, and so $I$ and $J$ are of the form $I=\operatorname{ker}\left(\operatorname{ind}_{G_{\mu}}^{G} \sigma\right)$ and $J=\operatorname{ker}\left(\operatorname{ind}_{G_{\mu}}^{G} \tau\right)$ for 
certain characters $\sigma, \tau$ extending $\lambda$. Now

$$
\chi_{\alpha} \cdot I=\operatorname{ker}\left(\chi_{\alpha} \otimes \operatorname{ind}_{G_{\mu}}^{G} \sigma\right)=\operatorname{ker}\left(\operatorname{ind}_{G_{\mu}}^{G}\left(\chi_{\alpha} \mid G_{\mu} \cdot \sigma\right)\right) \rightarrow \operatorname{ker}\left(\operatorname{ind}_{G_{\mu}}^{G} \tau\right),
$$

and hence $\left(\chi_{\alpha} \mid G_{\mu}\right) \cdot \sigma \rightarrow \tau$ in $\hat{G}_{\mu}$. Since $\chi_{\alpha} \mid L \equiv 1$ for all $\alpha$, we get that $\sigma|L=\tau| L$ and hence $\tau=\sigma \eta$ for some $\eta \in \widehat{G_{\mu} / L}$. Now choose any $\chi \in \widehat{G / L}$ extending $\eta$. Then, as required,

$$
J=\operatorname{ker}\left(\operatorname{ind}_{G_{\mu}}^{G}(\sigma \eta)\right)=\operatorname{ker}\left(\chi \otimes \operatorname{ind}_{G_{\mu}}^{G} \sigma\right)=\chi \cdot I \in \widehat{G / L} \cdot I .
$$

Theorem 4.2 answers the question of when the quotient map from $\operatorname{Prim}\left(C^{*}(G)\right)$ onto $\operatorname{Glimm}\left(C^{*}(G)\right)$ is open.

Theorem 4.2. For a two-step nilpotent locally compact group $G$ with centre $Z$ the following two conditions are equivalent.

(i) The map $\lambda \rightarrow F_{\lambda}$ from $\hat{Z}$ into $\mathcal{K}(G)$ is continuous.

(ii) The quotient map from $\operatorname{Prim}\left(C^{*}(G)\right)$ onto $\operatorname{Glimm}\left(C^{*}(G)\right)$ is open.

Proof. To prove that (i) implies (ii), suppose that there is an open subset $V$ of $\operatorname{Prim}\left(C^{*}(G)\right)$ such that $\operatorname{Sat}(V)$, the saturation of $V$ with respect to $\sim$, fails to be open. Then there exists $P \in \operatorname{Sat}(V)$ and a net $\left(P_{\alpha}\right)_{\alpha}$ in $\operatorname{Prim}\left(C^{*}(G)\right) \backslash \operatorname{Sat}(V)$ converging to $P$ in $\operatorname{Prim}\left(C^{*}(G)\right)$. With $\lambda_{\alpha}=r\left(P_{\alpha}\right)$ and $\lambda=r(P)$, we get that $\lambda_{\alpha} \rightarrow \lambda$ in $\hat{Z}$ and hence, by hypothesis, that $F_{\lambda_{\alpha}} \rightarrow F_{\lambda}$ in $\mathcal{K}(G)$. This implies that $\widehat{G / F_{\lambda_{\alpha}}} \rightarrow \widehat{G / F_{\lambda}}$ in $\mathcal{K}(\widehat{G / Z})$.

Now, since $P \in \operatorname{Sat}(V)$, there exists $\chi \in \widehat{G / F_{\lambda}}$ such that $\chi \cdot P \in V$ (Lemma 3.1). As $\widehat{G / F_{\lambda_{\alpha}}} \rightarrow \widehat{G / F_{\lambda}}$, for each $\alpha$ there exists $\chi_{\alpha} \in \widehat{G / F_{\lambda_{\alpha}}}$ such that $\chi_{\alpha} \rightarrow \chi$. It follows that $\chi_{\alpha} \cdot P_{\alpha} \rightarrow \chi \cdot P$. Since $V$ is open, $\chi_{\alpha} \cdot P_{\alpha} \in V$ and hence $P_{\alpha} \in \operatorname{Sat}(V)$ eventually. This is a contradiction.

Conversely, suppose that (ii) holds. Since $\mathcal{K}(G)$ is compact, the map $\mu \rightarrow F_{\mu}$ is continuous at $\lambda$ if for every net $\left(\lambda_{\alpha}\right)_{\alpha}$ converging to $\lambda$, whenever $F_{\lambda_{\alpha}} \rightarrow H$ for some $H \in \mathcal{K}(G)$, then $H=F_{\lambda}$. Notice that by Lemma 3.5, if $F_{\lambda_{\alpha}} \rightarrow H$ and $\lambda_{\alpha} \rightarrow \lambda$, then $H \supseteq F_{\lambda}$. By way of contradiction, suppose there is a net $\left(\lambda_{\alpha}\right)_{\alpha}$ in $\hat{Z}$ such that $\lambda_{\alpha} \rightarrow \lambda$ and $F_{\lambda_{\alpha}} \rightarrow H$ with $F_{\lambda}$ properly contained in $H$.

Choose $P \in r^{-1}(\lambda)$. There exist $P_{\alpha} \in r^{-1}\left(\lambda_{\alpha}\right)$ such that $P_{\alpha} \rightarrow P$ in $\operatorname{Prim}\left(C^{*}(G)\right)$. Now let

$$
C=\bigcup_{\alpha} \widehat{G / F_{\lambda_{\alpha}}} \cdot P_{\alpha} \bigcup \widehat{G / H} \cdot P .
$$

We claim that $C$ is closed in $\operatorname{Prim}\left(C^{*}(G)\right)$. To that end, notice first that each of the sets $\widehat{G / F_{\lambda_{\alpha}}} \cdot P_{\alpha}$ and $\widehat{G / H} \cdot P$ is closed in $\operatorname{Prim}\left(C^{*}(G)\right)$. Indeed, this follows from Lemma 4.1, since $F_{\lambda_{\alpha}} \subseteq G_{\lambda_{\alpha}}$ and hence also $H=\lim _{\alpha} F_{\lambda_{\alpha}} \subseteq G_{\lambda}$. It remains to consider a net 
$\left(\chi_{\alpha_{\beta}} \cdot P_{\alpha_{\beta}}\right)_{\beta}$, converging to some $Q \in \operatorname{Prim}\left(C^{*}(G)\right)$, with the additional property that, for no $\beta_{0}$ is

$$
\chi_{\alpha_{\beta}} \cdot P_{\alpha_{\beta}} \in \widehat{G / F_{\lambda_{\alpha_{0}}}} \cdot P_{\alpha_{0}}
$$

for some $\alpha_{0}$ and all $\beta \geqslant \beta_{0}$. We need to show that then $Q \in \widehat{G / H} \cdot P$. It follows that $\lambda_{\alpha_{\beta}} \rightarrow \lambda$, whence $Q \in r^{-1}(\lambda)$. Let

$$
P_{\alpha}=\operatorname{ker}\left(\operatorname{ind}_{G_{\lambda_{\alpha}}}^{G} \varphi_{\alpha}\right), \quad P=\operatorname{ker}\left(\operatorname{ind}_{G_{\lambda}}^{G} \varphi\right) \quad \text { and } \quad Q=\operatorname{ker}\left(\operatorname{ind}_{G_{\lambda}}^{G} \psi\right),
$$

where $\varphi_{\alpha} \in \widehat{G_{\lambda_{\alpha}}}$ and $\varphi, \psi \in \widehat{G_{\lambda}}$. Since $P_{\alpha} \rightarrow P$ we can assume, after passing to a subnet if necessary, that

$$
\left(G_{\lambda_{\alpha_{\beta}}}, \varphi_{\alpha_{\beta}}\right) \rightarrow(L, \varphi \mid L)
$$

in $\mathcal{S}(G)$ for some subgroup $L$ of $G_{\lambda}$. Clearly, then $H=\lim _{\beta} F_{\lambda_{\alpha_{\beta}}} \subseteq L$ and

$$
\left(F_{\lambda_{\alpha_{\beta}}}, \varphi_{\alpha_{\beta}} \mid F_{\lambda_{\alpha_{\beta}}}\right) \rightarrow(H, \varphi \mid H)
$$

On the other hand, $\chi_{\alpha_{\beta}} \cdot P_{\alpha_{\beta}} \rightarrow Q$ and $\chi_{\alpha_{\beta}} \mid F_{\lambda_{\alpha_{\beta}}} \equiv 1$. Hence

$$
\left(F_{\lambda_{\alpha_{\beta}}}, \varphi_{\alpha_{\beta}} \mid F_{\lambda_{\alpha_{\beta}}}\right) \rightarrow(H, \psi \mid H)
$$

which yields that $\psi \varphi^{-1} \in \widehat{G_{\lambda} / H}$. Choose any $\chi \in \widehat{G / H}$ such that $\chi \mid G_{\lambda}=\psi \varphi^{-1}$. It follows that

$$
Q=\operatorname{ker}\left(\operatorname{ind}_{G_{\lambda}}^{G} \psi\right)=\operatorname{ker}\left(\operatorname{ind}_{G_{\lambda}}^{G}\left(\chi \mid G_{\lambda} \cdot \varphi\right)\right)=\operatorname{ker}\left(\chi \otimes \operatorname{ind}_{G_{\lambda}}^{G} \varphi\right)=\chi \cdot P .
$$

This finishes the proof that $C$ is closed in $\operatorname{Prim}\left(C^{*}(G)\right)$.

Finally, let $V=\operatorname{Prim}\left(C^{*}(G)\right) \backslash C$. Then $V$ is open in $\operatorname{Prim}\left(C^{*}(G)\right)$, but $\operatorname{Sat}(V)$ is not. In fact, since $F_{\lambda}$ is properly contained in $H,[P] \backslash \widehat{G / H} \cdot P$ is a non-empty subset of $V$ and, therefore,

$$
[P]=\operatorname{Sat}([P] \backslash \widehat{G / H} \cdot P) \subseteq \operatorname{Sat}(V)
$$

If $\operatorname{Sat}(V)$ were open, then $P_{\alpha}$ would eventually belong to $\operatorname{Sat}(V)$, contradicting the fact that $\left[P_{\alpha}\right]=\widehat{G / F_{\lambda_{\alpha}}} \cdot P_{\alpha} \subseteq C$ for all $\alpha$. The quotient map is, therefore, not open, and this contradiction proves that (ii) implies (i).

The proof of the next lemma also makes substantial use of the properties of continuity of induction and continuity of restriction.

Lemma 4.3. Let $\lambda \in \hat{Z}$ and $\tau \in \hat{F}_{\lambda}$ be such that $\tau \mid Z=\lambda$. Then $\operatorname{ker}\left(\operatorname{ind}_{F_{\lambda}}^{G} \tau\right)$ is a primal ideal of $C^{*}(G)$ if and only if there exists a net $\left(\lambda_{\alpha}\right)_{\alpha}$ in $\hat{Z}$ such that $\lambda_{\alpha} \rightarrow \lambda$ in $\hat{Z}$ and $G_{\lambda_{\alpha}} \rightarrow F_{\lambda}$ in $\mathcal{K}(G)$. 
Proof. Let $I=\operatorname{ker}\left(\operatorname{ind}_{F_{\lambda}}^{G} \tau\right)$, and suppose first that such a net in $\hat{Z}$ exists. Then, since $\lambda_{\alpha} \rightarrow \lambda$ and $G_{\lambda_{\alpha}} \rightarrow F_{\lambda}$, there exist $\varphi_{\alpha} \in \hat{G}_{\lambda_{\alpha}}$ such that $\varphi_{\alpha} \mid Z=\lambda_{\alpha}$ and $\left(G_{\lambda_{\alpha}}, \varphi_{\alpha}\right) \rightarrow$ $\left(F_{\lambda}, \tau\right)$ in $\mathcal{S}(G)$ (Theorem 2.6). It follows that $P_{\alpha}=\operatorname{ker}\left(\operatorname{ind}_{G_{\lambda_{\alpha}}}^{G} \varphi_{\alpha}\right) \rightarrow P$ for every $P \in h(I)$, whence $I$ is a primal ideal.

Conversely, let $I$ be primal and let $\left(P_{\alpha}\right)_{\alpha}$ be a net in $\operatorname{Prim}\left(C^{*}(G)\right)$ converging to $P$ for every $P \in h(I)$. Let $\lambda_{\alpha}=r\left(P_{\alpha}\right)$ and $P_{\alpha}=\operatorname{ker}\left(\operatorname{ind}_{G_{\lambda_{\alpha}}}^{G} \varphi_{\alpha}\right)$, as usual. Then $\lambda_{\alpha} \rightarrow \lambda$ and after passing to a subnet if necessary, we can assume that $G_{\lambda_{\alpha}} \rightarrow H$ for some $H \subseteq \mathcal{K}(G)$. Now, since

$$
\operatorname{ind}_{G_{\lambda_{\alpha}}}^{G} \varphi_{\alpha} \rightarrow \operatorname{ind}_{F_{\lambda}}^{G} \tau \text { and } \quad\left(\operatorname{ind}_{G_{\lambda_{\alpha}}}^{G} \varphi_{\alpha}\right) \mid G_{\lambda_{\alpha}} \sim \varphi_{\alpha}
$$

the theorem on continuity of restriction implies that $\left(G_{\lambda_{\alpha}}, \varphi_{\alpha}\right) \rightarrow(H, \varphi)$ for every character $\varphi$ of $H$ that is weakly contained in $\left(\operatorname{ind}_{F_{\lambda}}^{G} \tau\right) \mid H$. Since obviously $F_{\lambda} \subseteq H$,

$$
\left(\operatorname{ind}_{F_{\lambda}}^{G} \tau\right) \mid H \sim\left\{\varphi \in \hat{H}: \varphi \mid F_{\lambda}=\tau\right\} .
$$

Thus there can be only one such $\varphi$. Equivalently, $H=F_{\lambda}$ and hence $G_{\lambda_{\alpha}} \rightarrow F_{\lambda}$ in $\mathcal{K}(G)$, as was to be shown.

Since a $C^{*}$-algebra $A$ is quasi-standard if and only if every Glimm ideal of $A$ is primal and the quotient map $q: \operatorname{Prim}(A) \rightarrow \operatorname{Glimm}(A)$ is open [3, Theorem 3.2], the following corollary is an immediate consequence of Theorem 4.2 and Lemma 4.3.

Corollary 4.4. Let $G$ be a two-step nilpotent locally compact group and $Z$ its centre. Then $C^{*}(G)$ is quasi-standard if and only if the following two conditions are satisfied.

(i) The map $\lambda \rightarrow F_{\lambda}$ from $\hat{Z}$ into $\mathcal{K}(G)$ is continuous.

(ii) For each $\lambda \in \hat{Z}$ there exists a net $\left(\lambda_{\alpha}\right)_{\alpha}$ in $\hat{Z}$ such that $\lambda_{\alpha} \rightarrow \lambda$ in $\hat{Z}$ and $G_{\lambda_{\alpha}} \rightarrow F_{\lambda}$ in $\mathcal{K}(G)$.

Remark 4.5. Suppose that $G$ is a simply connected two-step nilpotent Lie group with the property that every Glimm ideal of $C^{*}(G)$ is primal. Then $C^{*}(G)$ is quasi-standard [2, Theorem]. Equivalently, condition (ii) of Corollary 4.4 already ensures condition (i). It is unlikely that this same conclusion holds true for arbitrary locally compact two-step nilpotent groups. However, we are unaware of a counterexample. On the other hand, it is worth pointing out that there are three-step nilpotent simply connected Lie groups $G$ such that every Glimm ideal of $C^{*}(G)$ is primal, yet nevertheless $C^{*}(G)$ fails to be quasi-standard (see [2, Proposition 4] and [4]).

\section{The Glimm class of a primitive ideal}

For an arbitrary locally compact group $G$, let $\mathrm{FC}^{-}(G)$ denote the normal subgroup consisting of all elements with relatively compact conjugacy classes. If $G=\mathrm{FC}^{-}(G)$, 
then $\operatorname{Prim}\left(C^{*}(G)\right)$ is a Hausdorff space [16], and, for connected groups, the converse is true $[\mathbf{7}]$. Thus it is not surprising that, when studying the extent to which the Hausdorff property fails for $\operatorname{Prim}\left(C^{*}(G)\right)$, the subgroup $\mathrm{FC}^{-}(G)$ of $G$ is of importance.

Tits [21] has shown that $\mathrm{FC}^{-}(G)$ is closed in $G$ provided that $G$ is connected. However, it need not be closed in general $[\mathbf{2 1}]$. In passing we give an example of a two-step nilpotent group $G$ for which $\mathrm{FC}^{-}(G)$ fails to be closed.

Example 5.1. Let $C$ be the compact group $\left(\mathbb{Z}_{2}\right)^{\mathbb{Z}}$, and let

$$
D=\left\{\left(x_{n}\right)_{n} \in C: x_{n}=0 \text { for all } n \leqslant n_{x} \text { for some } n_{x} \in \mathbb{Z}\right\}
$$

endowed with the topology that makes the subgroups $D_{k}, k \in \mathbb{Z}$, with

$$
D_{k}=\left\{\left(x_{n}\right)_{n} \in D: x_{n}=0 \text { for all } n \leqslant k\right\},
$$

a neighbourhood basis of the identity. Let $G$ denote the group of upper triangular matrices

$$
\left(\begin{array}{lll}
1 & x & y \\
0 & 1 & z \\
0 & 0 & 1
\end{array}\right),
$$

where $x \in C$ and $y, z \in D$. It is then easily verified that such a matrix belongs to $\mathrm{FC}^{-}(G)$ if and only $x \in D$. Hence $\mathrm{FC}^{-}(G)$ is a proper dense subgroup of $G$.

From now on, let $G$ be a two-step nilpotent locally compact group. Note that, in this case, $x \in \mathrm{FC}^{-}(G)$ if and only if the subgroup $[G, x]$ of $Z$ is relatively compact. For every compact subgroup $K$ of $Z$, let $G(K)=\{x \in G:[x, G] \subseteq K\}$. Then $\mathrm{FC}^{-}(G)=\bigcup\{G(K)$ : $K$ a compact subgroup of $Z\}$. In particular, if $G$ is compact free (that is, no non-trivial element of $G$ generates a relatively compact subgroup), then $\mathrm{FC}^{-}(G)$ equals the centre of $G$.

Lemma 5.2. Let $N=\overline{F C^{-}(G)}$ and $\pi \in \hat{G}$. Then

$$
[\operatorname{ker} \pi] \subseteq h\left(\operatorname{ker}\left(\operatorname{ind}_{N}^{G}(\pi \mid N)\right)\right)
$$

Proof. Let $P=\operatorname{ker} \pi$ and $\lambda=r(P)$, and consider any $Q \in[P]$. Then $r(Q)=\lambda$, and hence there are characters $\sigma$ and $\tau$ of $G_{\lambda}$ extending $\lambda$ such that $P=\operatorname{ker}\left(\operatorname{ind}_{G_{\lambda}}^{G} \sigma\right)$ and $Q=\operatorname{ker}\left(\operatorname{ind}_{G_{\lambda}}^{G} \tau\right)$. Since $G / N$ is abelian,

$$
\operatorname{ind}_{G_{\lambda}}^{G} \tau \prec \operatorname{ind}_{N}^{G}\left(\left(\operatorname{ind}_{G_{\lambda}}^{G} \tau\right) \mid N\right) .
$$

Moreover, by Lemma 2.2, since $\tau$ is $G$-invariant,

$$
\left(\operatorname{ind}_{G_{\lambda}}^{G} \tau\right) \mid N \sim \operatorname{ind}_{G_{\lambda} \cap N}^{N}\left(\tau \mid G_{\lambda} \cap N\right)
$$


and similarly for $\sigma$. Therefore it suffices to verify that

$$
\operatorname{ind}_{G_{\lambda} \cap N}^{N}\left(\tau \mid G_{\lambda} \cap N\right) \sim \operatorname{ind}_{G_{\lambda} \cap N}^{N}\left(\sigma \mid G_{\lambda} \cap N\right),
$$

which is equivalent to $\tau\left|G_{\lambda} \cap N=\sigma\right| G_{\lambda} \cap N$. Of course, it is enough to show that $\tau\left|G_{\lambda} \cap G(K)=\sigma\right| G_{\lambda} \cap G(K)$ for every compact subgroup $K$ of $Z$. Now, fix $K$ and let

$$
L=G_{\lambda} \cap G(K) \quad \text { and } \quad M=K_{\lambda} \cap K .
$$

Since $[L, G] \subseteq K_{\lambda} \cap K, L / M$ is contained in the centre of $G / M$. Notice next that, since $\tau|M=\sigma| M=1, P$ and $Q$ are elements of $\operatorname{Prim}\left(C^{*}(G / M)\right)$, and since $M$ is compact, $\operatorname{Prim}\left(C^{*}(G / M)\right)$ is open and closed in $\operatorname{Prim}\left(C^{*}(G)\right)$. Thus $P$ and $Q$ cannot be separated by a continuous function on $\operatorname{Prim}\left(C^{*}(G / M)\right)$. Finally, since $L / M$ is contained in the centre of $G / M$, we obtain that $\tau|L=\sigma| L$, as required. This finishes the proof of the lemma.

It can happen that, for $\pi \in \hat{G}$, the inclusion of Lemma 5.2 is strict. Examples can be found within the class of two-step nilpotent simply connected Lie groups (see [4] and Example 6.3 below). However, it is not unlikely that for the trivial representation $1_{G}$ we always have $\left[\operatorname{ker} 1_{G}\right]=h\left(\operatorname{ker}\left(\operatorname{ind}_{N}^{G} 1_{N}\right)\right)=\operatorname{Prim}\left(C^{*}(G / N)\right)$. Such a conjecture is strongly supported by the partial results that follow. In the sequel we shall always identify the dual group $\widehat{G / Z(G)}$ with the corresponding closed subset of $\operatorname{Prim}\left(C^{*}(G)\right)$ and write $\left[1_{G}\right]$ for $\left[\operatorname{ker} 1_{G}\right]$.

Lemma 5.3. Let $\mathcal{H}$ be a system of open subgroups of $G$ each of which contains the centre, and suppose that $\mathcal{H}$ is upwards directed by inclusion and satisfies

$$
\bigcup\{H: H \in \mathcal{H}\}=G .
$$

If $\left[1_{\mathrm{H}}\right]=\widehat{H / Z(H)}$ for all $H \in \mathcal{H}$, then $\left[1_{G}\right]=\widehat{G / Z(G)}$.

Proof. Let $Z=Z(G)$, and for $H_{0} \in \mathcal{H}$ set

$$
X_{H_{0}}=\bigcup\left\{\widehat{G / H}: H \in \mathcal{H}, H_{0} \subseteq H\right\} .
$$

By the hypotheses on $\mathcal{H}, X_{H_{0}}$ is a subgroup of $\widehat{G / Z}$ and

$$
\bigcap\left\{Z(H): H_{0} \subseteq H \in \mathcal{H}\right\}=Z .
$$

Thus $X_{H_{0}}$ separates the points of $G / Z$ and hence is dense in $\widehat{G / Z}$.

Let $S$ denote the open subset of $\widehat{G / Z}$ consisting of all points of $\widehat{G / Z}$ that can be separated from $1_{G}$ by a continuous function on $\operatorname{Prim}\left(C^{*}(G)\right)$. We are going to prove that $S \cap X_{H_{0}}=\emptyset$ for some $H_{0} \in \mathcal{H}$. Since $X_{H_{0}}$ is dense in $\widehat{G / Z}$, this will show that $S=\emptyset$, as required. 
For each $H \in \mathcal{H}$, let $\mu_{\mathrm{H}}$ denote normalized Haar measure on the compact subgroup $\widehat{G / H}$ of $\widehat{G / Z}$. Now, since the mapping $(\chi, Q) \rightarrow \chi \cdot Q$ from $\widehat{G / Z} \times \operatorname{Prim}\left(C^{*}(G)\right)$ onto $\operatorname{Prim}\left(C^{*}(G)\right)$ is continuous, to every continuous function $f$ on $\operatorname{Prim}\left(C^{*}(G)\right)$ we can associate a function $f_{\mathrm{H}}$ on $\operatorname{Prim}\left(C^{*}(G)\right)$ defined by

$$
f_{\mathrm{H}}(P)=\int_{\widehat{G / H}} f(\chi \cdot P) \mathrm{d} \mu_{\mathrm{H}}(\chi) .
$$

Using the continuity property again and the fact that $\widehat{G / H}$ is compact, we see, easily, that $f_{\mathrm{H}}$ is continuous. Moreover, since $\widehat{G / H} \rightarrow\left\{1_{G}\right\}$ in $\mathcal{K}(\hat{G})$ as $H \rightarrow G$, it follows that $f_{\mathrm{H}}$ converges to $f$ pointwise on $\operatorname{Prim}\left(C^{*}(G)\right)$ as $H \rightarrow G$.

Now, let $\chi \in S$ and fix a continuous function $f$ on $\operatorname{Prim}\left(C^{*}(G)\right)$ such that $f(\chi) \neq$ $f\left(1_{G}\right)$. By what we have shown above there exists $H_{0} \in \mathcal{H}$ such that $f_{\mathrm{H}}(\chi) \neq f_{\mathrm{H}}\left(1_{G}\right)$ for all $H \in \mathcal{H}$ containing $H_{0}$. We claim that for every such $H, \chi \mid H$ can be separated from $1_{\mathrm{H}}$ by a continuous function on $\operatorname{Prim}\left(C^{*}(H)\right)$. To that end, recall first that the action of $G$ on $H$ by conjugation gives rise to an action $(x, Q) \rightarrow Q^{x}$ of $G$ on $\operatorname{Prim}\left(C^{*}(H)\right)$, and let $\operatorname{Prim}\left(C^{*}(H)\right) / G$ denote the space of all ideals $I$ of $C^{*}(H)$ of the form $I=\cap\left\{Q^{x}: x \in G\right\}$, where $Q \in \operatorname{Prim}\left(C^{*}(H)\right)$. The ideals in $\operatorname{Prim}\left(C^{*}(H)\right) / G$ are precisely the maximal closed $G$-invariant ideals of $C^{*}(H)$. Endow $\operatorname{Prim}\left(C^{*}(H)\right) / G$ with the hull-kernel topology. Then we have continuous mappings

$$
s: \operatorname{Prim}\left(C^{*}(H)\right) \rightarrow \operatorname{Prim}\left(C^{*}(H)\right) / G,
$$

defined by $s(Q)=\cap\left\{Q^{x}: x \in G\right\}$ for $Q \in \operatorname{Prim}\left(C^{*}(H)\right)$, and

$$
t: \operatorname{Prim}\left(C^{*}(G)\right) \rightarrow \operatorname{Prim}\left(C^{*}(H)\right) / G,
$$

by $t(P)=P \cap C^{*}(H)$ for $P \in \operatorname{Prim}\left(C^{*}(G)\right)$. Since $\widehat{G / H}$ is compact and $\operatorname{Prim}\left(C^{*}(G)\right)$ is a $T_{1}$-space, $\widehat{G / H} \cdot P$ is closed in $\operatorname{Prim}\left(C^{*}(G)\right)$ and hence equal to $t^{-1}(t(P))$ for every $P \in \operatorname{Prim}\left(C^{*}(G)\right)$. In particular, for every open subset $V$ of $\operatorname{Prim}\left(C^{*}(G)\right), t^{-1}(t(V))=$ $\widehat{G / H} \cdot V$ is open, and this implies that $t$ is actually open. Since $f_{\mathrm{H}}$ is constant on $\widehat{G / H} \cdot P$ for every $P \in \operatorname{Prim}\left(C^{*}(G)\right.$ ), we can therefore define a function $g_{\mathrm{H}}$ on $\operatorname{Prim}\left(C^{*}(H)\right) / G$ by setting $g_{\mathrm{H}}(t(P))=f_{\mathrm{H}}(P)$, and $g_{\mathrm{H}}$ is continuous. Then $g_{\mathrm{H}} \circ s$ is a continuous function on $\operatorname{Prim}\left(C^{*}(H)\right)$ satisfying

$$
g_{\mathrm{H}} \circ s(\chi \mid H)=g_{\mathrm{H}}(t(\chi))=f_{\mathrm{H}}(\chi) \neq f_{\mathrm{H}}\left(1_{G}\right)=g_{\mathrm{H}} \circ s\left(1_{\mathrm{H}}\right) .
$$

This proves the claim.

Finally, by hypothesis, $\left[1_{\mathrm{H}}\right]=\widehat{H / Z(H)}$. Thus we have shown that

$$
\chi \notin \bigcup\left\{\widehat{G / Z(H)}: H \in \mathcal{H}, H \supseteq H_{0}\right\},
$$

and this finishes the proof of the lemma. 
Recall that an element of a topological group is called compact if the closure of the cyclic group it generates is compact. A locally compact group $G$ is said to be compact-free if the unit element is the only compact element of $G$. These notions generalize those of a torsion element and torsion-freeness for discrete groups. If $G$ is nilpotent, then the set $G^{\mathrm{c}}$ of all compact elements of $G$ forms a closed normal subgroup of $G, G / G^{\mathrm{c}}$ is compact-free, and $G^{\mathrm{c}}$ is compact whenever $G$ is compactly generated [13].

The proof of the following lemma imitates an argument from [20, Lemma 2.5], where it has been used to determine the support in $\hat{G}$ of the conjugation representation of $G$ on $L^{2}(G)$ for a compactly generated, compact-free, two-step nilpotent group.

Lemma 5.4. Suppose that $G$ has a closed central subgroup $N$ such that $[G, G] \subseteq N$ and $N$ is of the form $N=\mathbb{R}^{p} \times \mathbb{Z}^{q}, p, q \in \mathbb{N}_{0}$. Then $\left[1_{G}\right]=\widehat{G / Z(G)}$.

Proof. Let $\lambda \in \widehat{Z(G)}$ and $\mu=\lambda \mid N$. There exist $\left(a_{1}, \ldots, a_{p}\right) \in \mathbb{R}^{p}$ and $\left(b_{1}, \ldots, b_{q}\right) \in$ $\mathbb{R}^{q}$ such that

$$
\mu(x, y)=\exp \left(2 \pi \mathrm{i}\left(\sum_{j=1}^{p} x_{j} a_{j}+\sum_{k=1}^{q} x_{k} b_{k}\right)\right)
$$

for all $x=\left(x_{1}, \ldots, x_{p}\right) \in \mathbb{R}^{p}$ and $y=\left(y_{1}, \ldots, y_{q}\right) \in \mathbb{Z}^{q}$. For $n \in \mathbb{N}$, define $\mu_{n} \in \hat{N}$ by

$$
\mu_{n}(x, y)=\exp \left(2 \pi \mathrm{i}\left(\frac{1}{2^{n}}\left(\sum_{j=1}^{p} x_{j} a_{j}+\sum_{k=1}^{q} y_{k} b_{k}\right)\right)\right) .
$$

Then $\mu_{n} \rightarrow 1_{N}$, and therefore we can extend $\mu_{n}$ to a character $\lambda_{n}$ of $Z(G)$ such that $\lambda_{n} \rightarrow 1_{Z(G)}$ as $n \rightarrow \infty$. Now

$$
K_{\lambda_{n}} \cap N=\left\{(x, y): \sum_{j=1}^{p} x_{j} a_{j}+\sum_{k=1}^{q} y_{k} b_{k} \in 2^{n} \mathbb{Z}\right\},
$$

and hence, since $[G, G] \subseteq N$,

$$
G_{\lambda_{n}}=\left\{u \in G:[u, G] \subseteq K_{\lambda_{n}} \cap N\right\} \subseteq G_{\lambda_{n-1}} \subseteq G_{\lambda}
$$

for all $n \geqslant 2$. It follows that the sequence $\left(G_{\lambda_{n}}\right)_{n}$ converges in $\mathcal{K}(G)$ to

$$
H=\bigcap_{n=1}^{\infty} G_{\lambda_{n}} \subseteq G_{\lambda} .
$$

By Theorem 2.6, for every $n \in \mathbb{N}$, we find a character $\varphi_{n}$ of $G_{\lambda_{n}}$ such that $\varphi_{n} \mid Z(G)=\lambda_{n}$ and $\left(G_{\lambda_{n}}, \varphi_{n}\right) \rightarrow\left(H, 1_{\mathrm{H}}\right)$ in $\mathcal{S}(G)$. By continuity of inducing,

$$
\operatorname{ind}_{G_{\lambda_{n}}}^{G} \varphi_{n} \rightarrow \operatorname{ind}_{\mathrm{H}}^{G} 1_{\mathrm{H}}
$$


and hence the sequence $\left(P_{n}\right)_{n}$, where $P_{n}=\operatorname{ker}\left(\operatorname{ind}_{G_{\lambda_{n}}}^{G}, \varphi_{n}\right)$ for $n \in \mathbb{N}$, converges in $\operatorname{Prim}\left(C^{*}(G)\right)$ to every point in $\widehat{G / H}$. Since $H \subseteq G_{\lambda}$ it follows that $\widehat{G / G_{\lambda}} \subseteq\left[1_{G}\right]$. Finally, since $\bigcap\left\{G_{\lambda}: \lambda \in \widehat{Z(G)}\right\}=Z(G)$, the subgroup of $\widehat{G / Z(G)}$ generated by all $\widehat{G / G_{\lambda}}, \lambda \in$ $\widehat{Z(G)}$, is dense in $\widehat{G / Z(G)}$. This proves that $\left[1_{G}\right]=\widehat{G / Z(G)}$.

Theorem 5.5. Let $G$ be a two-step nilpotent locally compact group such that the closure of its commutator subgroup contains a maximal compact subgroup $K$. Let $G(K)=$ $\{x \in G:[x, G] \subseteq K\}$. Then

$$
\left[1_{G}\right]=\widehat{G / G(K)} .
$$

In particular, if $G$ is compact-free, then $\left[1_{G}\right]=\widehat{G / Z(G)}$.

Proof. Recall first that since $K$ is compact and normal, $\operatorname{Prim}\left(C^{*}(G / K)\right)$ is open and closed in $\operatorname{Prim}\left(C^{*}(G)\right)$. Hence the Glimm class of $1_{G}$ in $\operatorname{Prim}\left(C^{*}(G)\right)$ coincides with the Glimm class of $1_{G / K}$ in $\operatorname{Prim}\left(C^{*}(G / K)\right)$. Since $G(K) / K$ equals the centre of $G / K$, after passing to $G / K$, we can therefore assume that $G$ has a closed central subgroup $F$ such that $F$ is compact-free and $[G, G] \subseteq F$. It suffices to show that, in this situation, $\left[1_{G}\right]=\widehat{G / Z(G)}$.

To that end, let $\mathcal{H}$ denote the set of all open subgroups $H$ of $G$ such that $H$ contains $Z(G)$ and $H / Z(G)$ is compactly generated. We claim that $\left[1_{\mathrm{H}}\right]=\widehat{H / Z(H)}$ for every $H \in \mathcal{H}$. Once this is shown, an application of Lemma 5.3 yields that $\left[1_{G}\right]=\widehat{G / Z(G)}$.

Now, being compact free, $F$ is of the form $F=\mathbb{R}^{p} \times L, p \in \mathbb{N}_{0}$, where $L$ is discrete and torsion-free. Since $H / Z(H)$ is compactly generated, so is $[H, H] \subseteq F$. Therefore the closed commutator subgroup $\overline{H^{\prime}}$ of $H$ is contained in some closed subgroup of $F$ of the form $\mathbb{R}^{p} \times M$, where $M$ is a finitely generated subgroup of $L$ and hence isomorphic to $\mathbb{Z}^{q}$ for some $q \in \mathbb{N}_{0}$. Thus Lemma 5.4 applies to $H$ and establishes the claim.

Let $P \in \operatorname{Prim}\left(C^{*}(G)\right)$ and $\lambda=r(P)$. We call $P$ a Glimm point if its Glimm class $[P]$ equals the singleton $\{P\}$. Now, if $P=\operatorname{ker}\left(\operatorname{ind}_{G_{\lambda}}^{G} \varphi\right)$, then $k([P])=\operatorname{ker}\left(\operatorname{ind}_{F_{\lambda}}^{G}\left(\varphi \mid F_{\lambda}\right)\right)$. This implies that $P$ is a Glimm point if and only if $G_{\lambda}=F_{\lambda}$. However, this latter equality may be difficult to verify because in general $F_{\lambda}$ is not easily computable.

If $P$ is a Glimm point, then in particular $P$ is a separated point of the topological space $\operatorname{Prim}\left(C^{*}(G)\right)$, that is, $P$ can be separated from every distinct point in $\operatorname{Prim}\left(C^{*}(G)\right)$ by open sets. Conversely, suppose that $V$ is an open subset of $\operatorname{Prim}\left(C^{*}(G)\right)$ consisting of separated points. The argument of [9, Proposition 7] then shows that each point in $V$ is even a Glimm point. This fact, in conjunction with the following lemma, can be used to identify Glimm points in $\operatorname{Prim}\left(C^{*}(G)\right)$ (see [4] and Example 6.3).

Lemma 5.6. $P \in \operatorname{Prim}\left(C^{*}(G)\right)$ is a separated point of $\operatorname{Prim}\left(C^{*}(G)\right)$ if and only if the map $\lambda \rightarrow G_{\lambda}$ is continuous at $r(P)$. 
Proof. Let $P$ be a separated point, so that $\{P\}=h(P)$ is a maximal limit set in $\operatorname{Prim}\left(C^{*}(G)\right)$. Since $\mathcal{K}(G)$ is compact, it suffices to show that if $\left(\lambda_{\alpha}\right)_{\alpha}$ is a net in $\hat{Z}$ converging to $\lambda=r(P)$ and $G_{\lambda_{\alpha}} \rightarrow H$ in $\mathcal{K}(G)$ for some $H$, then $H=G_{\lambda}$. We know that $H \subseteq G_{\lambda}$, so suppose that $H$ is strictly contained in $G_{\lambda}$. Since $\lambda_{\alpha} \rightarrow \lambda$, there are $P_{\alpha} \in r^{-1}\left(\lambda_{\alpha}\right)$ such that $P_{\alpha} \rightarrow P$ in $\operatorname{Prim}\left(C^{*}(G)\right)$. Let $P_{\alpha}=\operatorname{ker}\left(\operatorname{ind}_{G_{\lambda_{\alpha}}}^{G} \varphi_{\alpha}\right)$ and $P=$ $\operatorname{ker}\left(\operatorname{ind}_{G_{\lambda}}^{G} \varphi\right)$. Then, by Theorem 2.6, after moving to a subnet if necessary, we can assume that $\left(G_{\lambda_{\alpha}}, \varphi_{\alpha}\right) \rightarrow(H, \varphi \mid H)$ in $\mathcal{S}(G)$. By continuity of inducing, $h\left(\operatorname{ker}\left(\operatorname{ind}_{\mathrm{H}}^{G}(\varphi \mid H)\right)\right)$ is a limit set in $\operatorname{Prim}\left(C^{*}(G)\right)$, containing $P$. However, since $H$ is a proper subgroup of $G_{\lambda}$, there exists $\psi \in \hat{G}_{\lambda}$ such that $\psi \neq \varphi$, but $\psi|H=\varphi| H$. Then $Q=\operatorname{ker}\left(\operatorname{ind}_{G_{\lambda}}^{G} \psi\right) \in$ $\operatorname{Prim}\left(C^{*}(G)\right), Q \neq P$ and $Q$ is a limit point of $\left(P_{\alpha}\right)_{\alpha}$, a contradiction.

Conversely, suppose that $P$ is not separated, and choose $Q \in \operatorname{Prim}\left(C^{*}(G)\right)$ such that $Q \neq P$ and $Q$ cannot be separated from $P$ by open subsets. Then $P, Q \in r^{-1}(\lambda)$ for some $\lambda \in \hat{Z}$, and hence $P=\operatorname{ker}\left(\operatorname{ind}_{G_{\lambda}}^{G} \varphi\right)$ and $Q=\operatorname{ker}\left(\operatorname{ind}_{G_{\lambda}}^{G} \psi\right)$ for certain $\varphi, \psi \in \widehat{G_{\lambda}}$ such that $\varphi|Z=\lambda=\psi| Z$. Since there is a net in $\operatorname{Prim}\left(C^{*}(G)\right)$ converging to both $P$ and $Q$, by Theorem 2.6 there are $\left(\lambda_{\alpha}, G_{\lambda_{\alpha}}, \varphi_{\alpha}\right) \in P$ such that $\lambda_{\alpha} \rightarrow \lambda$ in $\hat{Z}$ and $\left(G_{\lambda_{\alpha}}, \varphi_{\alpha}\right) \rightarrow(H, \rho)$ for some $(H, \rho) \in \mathcal{S}(G)$ such that $H \subseteq G_{\lambda}$ and $\varphi|H=\rho=\psi| H$. Since $\varphi \neq \psi$, we necessarily have that $H$ is properly contained in $G_{\lambda}$. This shows that $\mu \rightarrow G_{\mu}$ is discontinuous at $\lambda$.

\section{Examples}

We conclude the paper with three examples, two of which are Heisenberg type groups, while the third one are the universal two-step nilpotent simply connected Lie groups. At least for Examples 6.2 and 6.3 below, the description of $\operatorname{Glimm}\left(C^{*}(G)\right)$ as a set and of its topology requires the results of $\S 3$.

Example 6.1. Let $k$ be a locally compact field and $G$ the group of upper triangular matrices

$$
\left(\begin{array}{lll}
1 & x & z \\
0 & 1 & y \\
0 & 0 & 1
\end{array}\right),
$$

where $x, y, z \in k$. It is easy to check that $G_{\lambda}=Z$ for all $\lambda \in \hat{Z}, \lambda \neq 1_{Z}$. As for the real Heisenberg group it follows that $C^{*}(G)$ is quasi-standard and the mapping $\lambda \rightarrow \operatorname{ker}\left(\operatorname{ind}_{Z}^{G} \lambda\right)$ is a homeomorphism between $\hat{Z}$ and $\operatorname{Glimm}\left(C^{*}(G)\right)$.

Example 6.2. Let $p$ be a prime, $\Omega_{p}$ the $p$-adic number field and $\Delta_{p}$ the subring of $p$-adic integers. We first study the two-step nilpotent group $G$ of upper triangular matrices

$$
\left(\begin{array}{lll}
1 & x & z \\
0 & 1 & y \\
0 & 0 & 1
\end{array}\right),
$$


where $y, z \in \Omega_{p}$ and $x \in \Delta_{p}$. In what follows we write this matrix as a triple $(x, y, z)$, and denote by $N$ the abelian normal subgroup consisting of all elements $(0, y, z)$. Since $G / N$ is compact, $G$ is of type I and hence, with the usual identification, $\hat{G}=\operatorname{Prim}\left(C^{*}(G)\right)$. Our aim is to determine the Glimm ideal space of $C^{*}(G)$.

For $k \in \mathbb{Z}, p^{k} \Delta_{p}$ is a compact open subgroup of $\Omega_{p}$, and such groups are the only non-trivial closed subgroups of $\Omega_{p}$ (see $\left.[\mathbf{1 4},(10.16)(\mathrm{a})]\right)$. Note that

$$
p^{k} \Delta_{p} \supseteq p^{k+1} \Delta_{p}, \quad \bigcup_{k \in \mathbb{Z}} p^{k} \Delta_{p}=\Omega_{p} \quad \text { and } \bigcap_{k \in \mathbb{Z}} p^{k} \Delta_{p}=\{0\} .
$$

Fix a character $\chi$ of $\Omega_{p}$ with kernel $\Delta_{p}$, and for each $y \in \Omega_{p}$, define $\lambda_{y} \in \widehat{\Omega_{p}}$ by $\lambda_{y}(x)=$ $\chi(y x), x \in \Omega_{p}$. Then the mapping $y \rightarrow \lambda_{y}$ is a topological isomorphism between $\Omega_{p}$ and $\widehat{\Omega_{p}}$. We now compute $G_{\lambda_{y}}$ for $y \neq 0$. There is a unique $k \in \mathbb{Z}$ such that $y \in p^{k} \Delta_{p} \backslash p^{k+1} \Delta_{p}$. Then

$$
\begin{aligned}
K_{\lambda_{y}} & =\{(0,0, z) \subseteq G: \chi(y z)=1\} \\
& =\{0\} \times\{0\} \times y^{-1} \Delta_{p}=\{0\} \times\{0\} \times p^{-k} \Delta_{p},
\end{aligned}
$$

and this implies that

$$
\begin{aligned}
G_{\lambda_{y}} & =\left\{(x, y, z) \in G:[(x, y, z), G] \subseteq K_{\lambda_{y}}\right\} \\
& =\left\{(x, y, z) \in G:\left(0,0, x y^{\prime}-x^{\prime} y\right) \in K_{\lambda_{y}} \text { for all } x^{\prime} \in \Delta_{p} \text { and } y^{\prime} \in \Omega_{p}\right\} \\
& =\left\{(x, y, z) \in G: x \Omega_{p} \subseteq p^{-k} \Delta_{p} \text { and } y \Delta_{p} \subseteq p^{-k} \Delta_{p}\right\} \\
& =\left\{(0, y, z) \in G: y \in p^{-k} \Delta_{p}\right\}=\{0\} \times p^{-k} \Delta_{p} \times \Omega_{p} .
\end{aligned}
$$

Let $\Lambda_{k}=\left\{\lambda_{y}: y \in p^{k} \Delta_{p} \backslash p^{k+1} \Delta_{p}\right\}, k \in \mathbb{Z}$. Then $\widehat{\Omega_{p}} \backslash\left\{1_{\Omega_{p}}\right\}$ is the disjoint union of the open sets $\Lambda_{k}$. The above computation shows that the map $\mu \rightarrow G_{\mu}$ is constant on each set $\Lambda_{k}$. Moreover, if $\left(\lambda_{n}\right)_{n}$ is an arbitrary sequence in $\hat{Z} \backslash\left\{1_{Z}\right\}$ converging to $1_{Z}$, then $G_{\lambda_{n}} \rightarrow N$. Indeed, if $\lambda_{n}=\lambda_{y_{n}}$ with $y_{n} \in \Lambda_{k_{n}}$, then necessarily $k_{n} \rightarrow \infty$ and hence $G_{\lambda_{n}}=\{0\} \times p^{-k_{n}} \Delta_{p} \times \Omega_{p} \rightarrow N$ in $\mathcal{K}(G)$.

These facts now lead to the description of $\operatorname{Glimm}\left(C^{*}(G)\right)$. Indeed, by Lemma 5.6 and the remark preceding it, every point of $\hat{G} \backslash \widehat{G / Z}$ is a Glimm point and hence $F_{\lambda}=G_{\lambda}=$ $\{0\} \times p^{-k} \Delta_{p} \times \Omega_{p}$ for $\lambda \in \Lambda_{k}, k \in \mathbb{Z}$. Now let $\gamma \in \widehat{G / Z}$. It follows from Lemma 5.2 or from the fact, that the quotient space $\hat{N} / G$ is Hausdorff, that $[\gamma] \subseteq \gamma \cdot \widehat{G / N}$. On the other hand, if $\left(\lambda_{n}\right)_{n}$ is an arbitrary sequence in $\hat{Z} \backslash\left\{1_{Z}\right\}$ converging to $1_{Z}$, then $\left(F_{\lambda_{n}}, \gamma \mid F_{\lambda_{n}} \cdot \lambda_{n}\right) \rightarrow(N, \gamma \mid N)$ in $\mathcal{S}(G)$ and hence

$$
\operatorname{ind}_{F_{\lambda_{n}}}^{G}\left(\gamma \mid F_{\lambda_{n}} \cdot \lambda_{n}\right) \rightarrow \operatorname{ind}_{N}^{G}(\gamma \mid N)
$$

Thus $\gamma \cdot \widehat{G / N}$ is a limit set in $\hat{G}$, and this proves that $[\gamma]=\gamma \cdot \widehat{G / N}$. Therefore, as a set,

$$
\begin{aligned}
& \operatorname{Glimm}\left(C^{*}(G)\right)=\bigcup_{k \in \mathbb{Z}}\left\{\operatorname{ker}\left(\operatorname{ind}_{p^{-k} \Delta_{p} \times \Omega_{p}}^{G}(\alpha \lambda)\right): \lambda \in \Lambda_{k}, \alpha \in \widehat{p^{-k} \Delta p}\right\} \\
& \bigcup\left\{\operatorname{ker}\left(\operatorname{ind}_{N}^{G} \alpha\right): \alpha \in \widehat{N / Z}\right\}
\end{aligned}
$$


and the topology is given by Theorem 3.7. In particular, we have seen that conditions (i) and (ii) of Corollary 4.4 are satisfied, so that $C^{*}(G)$ is quasi-standard.

Example 6.3. For $n \geqslant 2$, let $\mathfrak{w}_{n}$ denote the two-step nilpotent real Lie algebra with basis $\left\{X_{1}, \ldots, X_{n}\right\} \cup\left\{Y_{i, j}: 1 \leqslant i<j \leqslant n\right\}$ and non-zero products $\left[X_{i}, X_{j}\right]=Y_{i, j}$. Note that $\mathfrak{w}_{2}$ is the Heisenberg Lie algebra. Let $W_{n}=\exp \mathfrak{w}_{n}$ be the associated simply connected Lie group, and denote by $Z_{n}$ and $\mathfrak{z}_{n}$ the centre of $W_{n}$ and $\mathfrak{w}_{n}$, respectively. These groups $W_{n}$ are universal in the sense that every two-step nilpotent simply connected Lie group is a quotient of some $W_{n}$. In $[4, \S 2]$, separation properties of $\hat{W}_{n}$ and quasi-standardness of $C^{*}\left(W_{n}\right)$ have been studied. In what follows we assume the reader to be familiar with the usual notation in Kirillov theory.

Suppose first that $n$ is even. Then the maximal coadjoint orbit dimension in $\mathfrak{w}_{n}^{*}$ equals $n=\operatorname{dim}_{\mathfrak{z}}^{\perp}\left[\mathbf{4}\right.$, Theorem 2.4]. It follows from [2, Lemma 1] that $C^{*}\left(W_{n}\right)$ is quasistandard and that the mapping $\lambda \rightarrow \operatorname{ker}\left(\operatorname{ind}_{Z_{n}}^{W_{n}} \lambda\right)$ is a homeomorphism between $\hat{Z}_{n}$ and $\operatorname{Glimm}\left(C^{*}\left(W_{n}\right)\right)$.

Now let $n$ be odd. This case requires a more detailed analysis. For

$$
f=\sum_{r=1}^{n} \alpha_{r} X_{r}^{*}+\sum_{1 \leqslant i<j \leqslant n} \beta_{i, j} Y_{i, j}^{*} \in \mathfrak{w}_{n}^{*},
$$

let $B_{f}$ denote the skew-symmetric $n \times n$ matrix with entries $-\beta_{i, j}$ for $1 \leqslant i<j \leqslant n$ and $T_{f}$ the linear mapping from $\mathbb{R}^{n}$ into $\mathfrak{z}_{n}^{\perp}$ with matrix $B_{f}$ relative to the standard basis in $\mathbb{R}^{n}$ and the basis $\left\{X_{1}^{*}, \ldots, X_{n}^{*}\right\}$ in $\mathfrak{z}_{n}^{\perp}$. Then [4, Lemma 2.3]

$$
\operatorname{Ad}^{*}\left(W_{n}\right) f=f+T_{f}\left(\mathbb{R}^{n}\right),
$$

and $\pi_{f}$ is a separated point of $\hat{W}_{n}$ (equivalently, $f$ has maximal coadjoint orbit dimension) if and only if $\operatorname{dim} T_{f}\left(\mathbb{R}^{n}\right)=n-1$. In particular, $C^{*}\left(W_{n}\right)$ fails to be quasi-standard [4, Theorem 2.4]. We identify $\mathfrak{z}_{n}^{*}$ with $\hat{Z}_{n}$ by the map $f \rightarrow \lambda_{f}$, where

$$
\lambda_{f}(x)=\exp 2 \pi \mathrm{i} f(\log x), \quad x \in Z_{n},
$$

and write $F_{f}$ and $G_{f}$ instead of $F_{\lambda_{f}}$ and $G_{\lambda_{f}}$, respectively. Let $U$ be the open subset of all $f \in \mathfrak{z}_{n}^{*}$ with $\operatorname{dim} T_{f}\left(\mathbb{R}^{n}\right)=n-1$. Then, for $f \in U$,

$$
F_{f}=G_{f}=\exp \left\{X \in \mathfrak{w}_{n}: g(X)=0 \text { for all } g \in T_{f}\left(\mathbb{R}^{n}\right)\right\} .
$$

It follows from Lemma 5.6 or can be seen directly that the mapping $f \rightarrow F_{f}=G_{f}$ from $U$ into $\mathcal{K}\left(W_{n}\right)$ is continuous. If $f \in \mathfrak{z}_{n}^{*} \backslash U$, then, for every $g \in f+\mathfrak{z}_{n}^{\perp}, \pi_{g}$ cannot be separated from $\pi_{f}$ in $\hat{W}_{n}\left[\mathbf{4}\right.$, Theorem 2.6]. Hence $F_{f}=Z_{n}$. Thus, as a set,

$$
\begin{aligned}
& \operatorname{Glimm}\left(C^{*}\left(W_{n}\right)\right)=\left\{\operatorname{ker}\left(\operatorname{ind}_{Z_{n}}^{W_{n}} \lambda_{f}\right): f \in \mathfrak{z}_{n}^{*}, f \text { non-generic }\right\} \\
& \qquad\left\{\operatorname{ker}\left(\operatorname{ind}_{F_{f}}^{W_{n}} \tau\right): \tau \in \widehat{F_{f}}, \tau \mid Z_{n}=\lambda_{f}, f \in \mathfrak{z}_{n}^{*} \text { generic }\right\} .
\end{aligned}
$$


The topology on $\operatorname{Glimm}\left(C^{*}\left(W_{n}\right)\right)$ can be described by Theorem 3.7 as follows. The first set on the right is closed in $\operatorname{Glimm}\left(C^{*}(G)\right)$ and homeomorphic to $\mathfrak{z}_{n}^{*} \backslash U$. The second set on the right is homeomorphic to the subset $\left\{\left(F_{f}, \tau\right): f \in U, \tau \in \hat{F}_{f}, \tau \mid Z_{n}=\lambda_{f}\right\}$ of $\mathcal{S}\left(W_{n}\right)$. Finally, a sequence $\left(\operatorname{ker}\left(\operatorname{ind}_{F_{l}}^{W_{n}} \tau_{l}\right)\right)_{l}$ converges to $\operatorname{ker}\left(\operatorname{ind}_{Z_{n}}^{W_{n}} \lambda_{f}\right), f \in \mathfrak{z}_{n}^{*} \backslash U$, if $f_{n} \rightarrow f$ in $\mathfrak{z}_{n}^{*}$ and the sequence $\left(F_{f_{l}}, \tau_{l}\right)_{l}$ is convergent in $\mathcal{S}\left(W_{n}\right)$.

Acknowledgements. This work is supported by the German Research Foundation (DFG) and the Australian Research Council (ARC).

\section{References}

1. R. J. Archbold, Topologies for primal ideals, J. Lond. Math. Soc. (2) 36 (1987), 524-542.

2. R. J. Archbold and E. Kaniuth, Simply connected nilpotent Lie groups with quasistandard $C^{*}$-algebras, Proc. Am. Math. Soc. 125 (1997), 2733-2742.

3. R. J. Archbold and D. W. B. Somerset, Quasi-standard $C^{*}$-algebras, Math. Proc. Camb. Phil. Soc. 107 (1990), 349-360.

4. R. J. Archbold, E. Kaniuth, G. Schlichting and D. W. B. Somerset, On the topology of the dual of a nilpotent Lie group, Math. Proc. Camb. Phil. Soc. 125 (1999), 269-293.

5. L. BAGGETT, A description of the topology on the dual spaces of certain locally compact groups, Trans. Am. Math. Soc. 132 (1968), 175-215.

6. L. BAgGetT And J. PACKer, The primitive ideal space of two-step nilpotent group $C^{*}$-algebras, J. Funct. Analysis 124 (1994), 389-426.

7. L. Baggett and T. Sund, The Hausdorff dual problem for connected groups, J. Funct. Analysis 43 (1981), 60-68.

8. J. DAUns And K. H. Hofmann, Representations of rings by continuous sections, Memoirs of the American Mathematical Society, vol. 83 (American Mathematical Society, 1968).

9. C. Delaroche, Sur les centres des $C^{*}$-algèbres, II, Bull. Sc. Math. France (2) 92 (1968), 111-128.

10. J. Dixmier, $C^{*}$-algebras (Amsterdam, North-Holland, 1977).

11. J. M. G. FELL, A Hausdorff topology on the closed subsets of a locally compact nonHausdorff space, Proc. Am. Math. Soc. 13 (1962), 472-476.

12. J. M. G. FELL, Weak containment and induced representations of groups, II, Trans. Am. Math. Soc. 110 (1964), 424-447.

13. V. M. GLUŠKOV, Locally nilpotent locally bicompact groups (in Russian), Trudy Moskov. Mat. Obšc. 4 (1955), 291-332.

14. E. HewitT AND K. A. Ross, Abstract harmonic analysis, vol. I (Springer 1963).

15. R. Howe, The Fourier transform for nilpotent locally compact groups, Pacific J. Math. 73 (1977), 307-327.

16. E. KaniUth, Primitive ideal spaces of groups with relatively compact conjugacy classes, Arch. Math. 32 (1979), 16-24.

17. E. Kaniuth, On primary ideals in group algebras, Monash Math. 93 (1982), 293-302.

18. E. Kaniuth, G. Schlichting And K. F. Taylor, Minimal primal and Glimm ideal spaces of group $C^{*}$-algebras, J. Funct. Analysis 130 (1995), 43-76.

19. R. LEE, Full algebras of operator fields trivial except at one point, Indiana Univ. Math. J. 26 (1977), 351-372.

20. A. MARKFORT, On the support of the conjugation representation for solvable locally compact groups, Forum Math. 6 (1994), 431-449.

21. J. Tits, Automorphismes à déplacement borné des groupes de Lie, Topology 3 (Suppl. 1) (1964), 97-107. 
22. D. P. Williams, Transformation group $C^{*}$-algebras with Hausdorff spectrum, Illinois J. Math. 26 (1982), 317-321.

23. D. P. Williams, The topology on the primitive ideal space of transformation group $C^{*}$-algebras, Trans. Am. Math. Soc. 266 (1981), 335-359. 